\title{
ALMOST PERIODIC FUNCTIONS, CONSTRUCTIVELY
}

\author{
BAS SPITTERS $^{a}$
}

Institute for Computing and Information Sciences, Radboud University of Nijmegen

e-mail address: spitters@cs.ru.nl

\begin{abstract}
Almost periodic functions form a natural example of a non-separable normed space. As such, it has been a challenge for constructive mathematicians to find a natural treatment of them. Here we present a simple proof of Bohr's fundamental theorem for almost periodic functions which we then generalize to almost periodic functions on general topological groups.
\end{abstract}

\section{INTRODUCTION}

Finding a natural constructive treatment of the theory of almost periodic functions has long been a challenge for constructive mathematics, see [2], [5] and [7]. The present approach is similar to the one by Bishop's student Brom [2]. However, we replace his long and explicit construction by a simple definition of a new metric on the group, due to von Neumann [11](p.447), and applying Fourier and Peter-Weyl theory. Thus we obtain a very similar, but much more conceptual construction.

By constructive mathematics we mean constructive in the sense of Bishop [1]. That is, using intuitionistic logic and an appropriate set theory, or type theory. Like Bishop, we will freely use the axiom of (countable) dependent choice.

To introduce the theory of almost periodic functions we first consider a periodic function $f: \mathbb{R} \rightarrow \mathbb{C}$ with period $2 \pi$, say. We may identify $f$ with a function $g$ on the unit circle, by defining $g\left(e^{i x}\right):=f(x)$, for all $x \in \mathbb{R}$. Because the circle is a compact Abelian group, Fourier theory may be used to approximate the periodic function $f$ by finite sums of characters.

The sum $\lambda x \cdot e^{i x}+e^{i 2 x}$ is periodic, and this remains true when 2 is replaced by any other rational number. However, the sum of the periodic functions $\lambda x \cdot e^{i x}$ and $\lambda x \cdot e^{i \sqrt{2} x}$ is not periodic. But this sum is almost periodic. A real function $f$ is almost periodic if for every $\varepsilon>0$, there exists $l>0$ such that every interval $[t, t+l]$ contains at least one number $y$ for which $|f(x)-f(x+y)|<\varepsilon$ whenever $x$ is in $\mathbb{R}$. Classically, the class of almost periodic functions is closed under addition and multiplication, but constructively this is not

2000 ACM Subject Classification: F.4.1.

Key words and phrases: Almost periodic functions, constructive mathematics, topological groups.

${ }^{a}$ The author was supported by the Netherlands Organization for Scientific Research (NWO).

\footnotetext{
${ }^{1}$ We use the notation $\lambda x . t$ for the function that returns $t$ on input $x$. An alternative notation would by $x \mapsto t$.
} 
the case. When $a \neq 0$ the function $\lambda x . e^{i a x}$ is periodic. When $a \downarrow 0$ the period tends to $\infty$. Consequently, when we do not know whether $a=0$ or $a \neq 0$, we are unable to show that $\lambda x . e^{i a x}$ is almost periodic. In [5] it is shown that the function $\lambda x . e^{i a x}$ is almost periodic if and only if $a=0$ or $a \neq 0$. Observe that if $a$ is close to 0 , then both $e^{i(1+a) x}$ and $e^{-i x}$ are almost periodic. However $e^{i(1+a) x} e^{-i x}=e^{i a x}$ is almost periodic if and only if $a=0$ or $a \neq 0$. Consequently, one can not prove constructively that the almost periodic functions are closed under multiplication. A similar argument shows that the almost periodic functions are not constructively closed under addition. It is, however, straightforward to prove that the almost periodic functions are closed under uniform limits.

\section{Preliminaries}

In this section we collect some results from constructive topological group theory, mostly following [1] [3]. We used [6], [10] and [8] as general references for the classical theory. In this section $G$ will denote a topological group.

Definition 1. A topological group is a group that is also a topological space in such a way that the group operations are continuous.

Any locally compact group allows a unique translation invariant measure, called Haar measure [1]. For a simple construction of Haar measure on compact groups see [3]. This construction follows von Neumann's classical existence proof.

Let $G$ be a compact group. Let $L_{1}$ denote $L_{1}(G)$, the space of Haar-integrable functions on $G$. Define the convolution from $L_{1} \times L_{1}$ to $L_{1}$ by

$$
f * g=\lambda x \cdot \int f(y) g\left(y^{-1} x\right) d y
$$

for all $f, g \in L_{1}$. This map is continuous, in fact $\|f * g\|_{1} \leq\|f\|_{1}\|g\|_{1}$. Define the involution $\tilde{f}:=\lambda x . f\left(x^{-1}\right)$. With this involution and convolution as multiplication $L_{1}$ is a $*$-algebra, called the group algebra. The group algebra contains much information about the group. For instance a group is Abelian if and only if its group algebra is Abelian. The group $G$ is compact, so its Haar measure is finite, consequently $L_{2}(G) \subset L_{1}(G)$, and thus, the convolution product $f * g$ belongs to $L_{2}$, for all $f \in L_{1}$ and $g \in L_{2}$. Moreover, $\|f * g\|_{2} \leq$ $\|f\|_{1}\|g\|_{2}$. Thus an element $f$ of $L_{1}$ can be considered as an operator $\lambda g . f * g$ on the Hilbert space $L_{2}$. These operators are compact, and thus normable, so $L_{1}$ can thus be completed to a $\mathrm{C}^{*}$-algebra. This allows us to use:

Theorem 1. [Gelfand] Let $\mathcal{A}$ be a unital commutative $\mathrm{C}^{*}$-algebra. The spectrum $X$ of $\mathcal{A}$ - that is, set of $\mathrm{C}^{*}$-algebra morphisms from $\mathcal{A}$ to $\mathbb{C}$ - can be equipped with a topology such that $X$ is compact and the Gelfand transform ${ }^{\wedge}: \mathcal{A} \rightarrow C(X)$, defined by $\hat{a}(x):=x(a)$, is a $\mathrm{C}^{*}$-isomorphism.

We will sometimes speak about the spectrum of a ${ }^{*}$-algebra when we mean the spectrum of the $\mathrm{C}^{*}$-algebra as constructed above.

Let $Z$ denote the center of the group algebra - that is the set of $f$ such that $f * g=g * f$ whenever $g$ is in $L_{1}$. A character is a $\mathrm{C}^{*}$-algebra morphism from $Z$ to $\mathbb{C}$. In case the group is Abelian, $Z=L_{1}$ and the characters are in one-one correspondence [1] (p.425) with continuous functions $\alpha: G \rightarrow \mathbb{C}$ such that $|\alpha(x)|=1$ and $\alpha(x y)=\alpha(x) \alpha(y)$, for all $x, y \in G$. Remaining in the Abelian case, the characters, with the usual multiplication of functions, form a group, denoted $G^{*}$. This group is called the dual group or character group. We equip the character group with the metric induced by the sup-norm $\|\cdot\|_{\infty}$. 
Theorem 2. 1] (Thm. 8.3.17) The character group $G^{*}$ of a locally compact Abelian group $G$ is a locally compact Abelian group.

Following [1] we define '(locally) compact space' to mean (locally) compact metric space. In fact, considering the more general case of a locally compact group, Bishop and Bridges introduce a new metric $\rho^{*}$ on $G^{*}$ such that $\left(G^{*}, \rho^{*}\right)$ is locally compact. For compact groups this metric is equivalent to the metric induced by the norm $\|\cdot\|_{\infty}$ on $C(G)$, see [1] (Lemma 8.3.16).

As a paradigm consider the Abelian group $G:=\left(\left\{e^{i t}: t \in \mathbb{R}\right\}, \cdot\right)$. The character group of this group is the space of the functions $\left\{\lambda z \cdot z^{n}: n \in \mathbb{Z}\right\} \subset C(G)$ with the metric and multiplication inherited from the normed space $C(G)$ of continuous functions on $G$. In this case $G$ is compact and $G^{*}$ is discrete. This is the general situation.

Theorem 3. Let $G$ be a compact Abelian group. Then the character group $G^{*}$ is discrete.

Proof. Let 1 be the constant function with value 1 . This function is a character. The set of characters $\alpha$ with $\|\alpha-1\|_{\infty}<1$ is an open set which contains only the character 1 . Indeed, if $\alpha(x) \neq 1$ for some $x \in G$, then for some $n, \alpha(x)^{n} \in\{z: \Re z \leq 0\}$. Since $\alpha(x)^{n}=\alpha\left(x^{n}\right)$, it follows that $\left|\alpha\left(x^{n}\right)-1\right| \geq 1$. Consequently, there is a neighborhood of the character 1 which contains only this character. By translation of this neighborhood one obtains for each element in the group $G^{*}$ a neighborhood containing only that element.

Every inhabited discrete separable metric space is countable, since any dense subset must coincide with the whole space. If $G$ is a compact group, then $G^{*}$ is locally compact, and hence separable, since moreover, $G^{*}$ is discrete, it is countable.

For a locally compact space $X$ let $C_{\infty}(X)$ denote the set of functions that are 'zero at infinity'. Bishop and Bridges [1] (p.431,p.442) proved the following Fourier theorem.

Theorem 4. Let $G$ be a locally compact Abelian group. There is a norm-decreasing linear map $\mathcal{F}$ from $L_{1}(G)$ to $C_{\infty}\left(G^{*}\right)$ such that $\mathcal{F}(f * g)=\mathcal{F}(f) \mathcal{F}(g)$ and $\mathcal{F}(f)=\lambda \alpha . \int f(x) \alpha(x) d x$ whenever $f, g$ are in $L_{1}(G)$. The map $\mathcal{F}$ is called the Fourier transform. Haar measure $\mu^{*}$ on $G^{*}$ can be normalized in such a way that $\mathcal{F}$ preserves the $L_{2}$-norm on $L_{1}(G) \cap L_{2}(G)$ and the map $\mathcal{F}^{*}: L_{1}\left(G^{*}\right) \rightarrow C_{\infty}(G)$ defined by $\mathcal{F}^{*}(\phi):=\lambda x \cdot \int \overline{\alpha(x)} \phi(\alpha) d \mu^{*}(\alpha)$ has the following property: $\mathcal{F}^{*} \mathcal{F} f=f$, for all $f \in L_{1}(G) \cap L_{2}(G)$.

In [3] the following results were proved for a general, not necessarily Abelian, compact group $G$. In this context $Z$ denotes the center of the group algebra and $\Sigma$ denotes a locally compact subset of its spectrum as a $\mathrm{C}^{*}$-algebra. The points of $\Sigma$ are called characters. We recall that $\Sigma$ is discrete.

We define the linear functional $I(f):=f(e)$ on the group algebra and remark that $f * g(e)=(f, \tilde{g})$, the inner product with respect to the Haar integral.

Theorem 5. Let $f$ be an element of $Z$ such that $\hat{f} \geq 0$. Let $a_{\sigma}:=\hat{f}(\sigma) /\left\|\chi_{\sigma}\right\|_{2}^{2}$ whenever $\sigma$ is an element of $\Sigma$. Then $\hat{f}(\sigma)=a_{\sigma} \widehat{\chi_{\sigma}}(\sigma), I(f)=\sum a_{\sigma}$ and $f=\sum a_{\sigma} \chi_{\sigma}$ uniformly.

Let $e_{\sigma}:=\chi_{\sigma} /\left\|\chi_{\sigma}\right\|_{2}$ and $b_{\sigma}(f):=\left(f, e_{\sigma}\right)$. Then $\left\|e_{\sigma}\right\|_{2}=1$ and $b_{\sigma}=\hat{f}(\sigma) /\left\|\chi_{\sigma}\right\|_{2}$.

Corollary 1. [Plancherel] For all $f$ in $Z, I(f * \tilde{f})=\sum\left|b_{\sigma}\right|^{2}$ and $e_{\sigma}$ is an orthonormal basis for the pre-Hilbert space $Z$.

The main theorem in the Peter-Weyl theory may be formulated as follows.

Theorem 6. For each $f \in C(G), \sum_{\sigma} e_{\sigma} * f$, where $\sigma \in \Sigma$, converges to $f$ in $L_{2}$. 
Usually, the Peter-Weyl theorem speaks about irreducible representations. Fortunately, these representations are in one-one correspondences with the characters above.

\section{Almost periodic Functions on Abelian groups}

In this section we will prove a constructive Bohr approximation theorem for the Abelian groups.

As is well-known, it is in general not possible to compute the norms of constructive analogues of non-separable normed spaces. Fortunately, there are, at least, two solutions to this problem: using quasi-norms, or using generalized real numbers. We repeat the definition from [1] (p.343).

Definition 2. Let $X$ be a linear space over a scalar field $F$, where either $F=\mathbb{R}$ or $F=\mathbb{C}$. A seminorm $\|\cdot\|$ is a map from $X$ to $\mathbb{R}$ such that for all $a \in F$ and $x, y \in X,\|x\| \geq 0$, $\|a x\|=|a|\|x\|$ and $\|x+y\| \leq\|x\|+\|y\|$. A quasinorm on $X$ is a family $\left\{\|\cdot\|_{i}: i \in I\right\}$ of seminorms on $X$ such that for each $x \in X$, the set $\left\{\|x\|_{i}: i \in I\right\}$ is bounded. Define the apartness relation $\neq$ on $X$ by $x \neq y$ if and only if there exists $i \in I$ such that $\|x-y\|_{i}>0$. Likewise, define the equality $x=y$ if and only if not $x \neq y$, for all $x, y \in X$. Then $\left(X,\left\{\|\cdot\|_{i}\right.\right.$ : $i \in I\})$ is called a quasinormed space.

A quasinormed space may also be viewed as a normed space where the norm is a generalized real number in the sense of Richman [4]. That is, the norm is a Dedekind cut in the real numbers, but this cut does not need to be located.

Although previous constructive developments considered only almost periodic functions defined on the real numbers it is natural to consider almost periodic functions over general groups. One of von Neumann's main ideas is that the almost periodic functions allow us to mimic most of the important constructions of compact groups. Most importantly, one can define a 'Haar measure' on the set of almost periodic functions. See [3] for a constructive proof of von Neumann's classical 'construction' of Haar measure on compact groups.

Here, instead of mimicking these constructions, we take a slightly different path: we define a new topology on $G$ and use the theory of compact groups directly. Classically, this would be slightly less general since we exclude non-continuous functions. Constructively, one can not define (total) non-continuous functions. So, like Loomis, we restrict ourselves to continuous functions.

Let $F$ denote either $\mathbb{R}$ or $\mathbb{C}$ and let $C_{b}(X, F)$ denote the bounded continuous $F$-valued functions on the set $X$. We will drop the field $F$ when it is either clear from the context, or irrelevant. The space $C_{b}(X)$ is a quasi-normed space with quasi-norm $\left\{\|\cdot\|_{x}: x \in X\right\}$, where $\|f\|_{x}$ is defined as $|f(x)|$.

A subset $A$ of a quasi-normed space is called totally bounded if for each $\varepsilon>0$, there is a finitely enumerable set $f_{1}, \ldots, f_{n} \in A$ such that for each $f \in A$, there exists $i$ such that $\left\|f-f_{i}\right\|_{j}<\varepsilon$ whenever $j$ is in $I$. Note that unlike in the metric case, we can not require all the elements $f_{1}, \ldots, f_{n}$ to be distinct.

Let $G$ be an Abelian group. Define the operator $T_{s}$ from $C_{b}(G)$ to $C_{b}(G)$ by $T_{s}=$ $\lambda f . \lambda x . f(s+x)$ for all $s$ in $G$.

Definition 3. A function $f \in C_{b}(G)$ is almost periodic if the set $S_{f}:=\{T(s) f: s \in G\}$ is a totally bounded subset of $C_{b}(G)$. 
In case $G=\mathbb{R}$, this definition is equivalent to Bohr's original definition, which we stated on p.1. Loomis' proof [6] (41F, p.171) of this fact is constructive. We note that every almost periodic function is uniformly continuous.

Let $f$ be an almost periodic function on $G$. The function $v_{a}:=\lambda g . g(a)$ is a uniformly continuous function from $C_{b}(G)$ to $G$. Because $S_{f}$ is totally bounded, we may define a pseudometric on $G$ by

$$
d_{f}(a, b):=\sup _{g \in S_{f}}\left|v_{a}(g)-v_{b}(g)\right|=\sup _{x \in G}|f(a+x)-f(b+x)| .
$$

This metric is invariant under the action of the group $(G,+)$, that is, $d_{f}(a+c, b+c)=$ $d_{f}(a, b)$, for all $a, b, c \in G$. Since $\left\|T_{y} f-T_{z} f\right\|_{\infty}=\sup _{x}|f(x+y)-f(x+z)|=d_{f}(y, z)$, there is an isometric embedding from $S_{f}$ into $\left(G, d_{f}\right)$. Consequently, $\left(G, d_{f}\right)$ is totally bounded and we let $G_{f}$ denote the completion of $\left(G, d_{f}\right)$, which is a compact group.

Recall from theorem 3 that the character group of a metric compact Abelian group is discrete. We obtain the following Plancherel theorem for almost periodic functions.

Theorem 7. Let $f$ be an almost periodic function. Let $\Sigma=G_{f}^{*}$ be the character group of the compact group $G_{f}$. Then $f$ is a continuous function on $G_{f}$ and $f=\sum \hat{f}(\sigma) \chi(\sigma)$ in $l_{2}(\Sigma)$.

The function $f$ is uniformly continuous. So for every $\varepsilon>0$, there is a $\delta>0$, such that when $|a-b|<\delta$, then $|f(a+x)-f(b+x)|<\varepsilon$ for all $x \in G$. Hence $d_{f}(a, b) \leq \varepsilon$. Consequently, any continuous function on $G_{f}$ is a continuous function on $G$, and thus characters of $G_{f}$ are characters of $G$. This shows that the space $\Sigma$ in the previous theorem is the canonical choice.

The following theorem is called the Bohr approximation theorem. As we remarked before, in constructive mathematics a sum, and therefore a linear combination, of characters need not be almost periodic.

Theorem 8. Let $f$ be an almost periodic function on $G$. Then $f$ can be approximated uniformly by an almost periodic linear combination of characters.

Proof. Let $I_{G}$ be a subset of $\mathbb{N}$ which is in bijective correspondence with $\Sigma$. Then $\Sigma=$ $\left\{\chi_{n}: n \in I_{G}\right\}$ and we define $I_{n}:=\left\{i \leq n: i \in I_{G}\right\}$. Let $P_{n}$ be the projection in $L_{2}$ on $\operatorname{span}\left\{\chi_{i}: i \in I_{n}\right\}$. Then $P_{n} f \rightarrow f$ uniformly as $n \rightarrow \infty$, by theorem 4.3 [3]. Since $P_{n}=\lambda f \cdot \sum_{i \in I_{n}} \chi_{i} * f$, it follows from equation (3.4) in [3] that $P_{n}$ commutes with $T_{s}$ whenever $s$ is in $G$. Consequently, $S_{P_{n} f}=\left\{T_{s} P_{n} f: s \in G\right\}=P_{n} S_{f}$. This set is totally bounded, because $S_{f}$ is totally bounded and $P_{n}$ is uniformly continuous. It follows that $P_{n} f$ is almost periodic.

The measure $\mu$ we used in Theorem [7, Haar measure on $G_{f}$, may seem a little ad hoc. In fact $\mu(f)$ is equal to the value of the unique constant function in closure of the convex hull of $S_{f}$. See the construction of Haar measure in [11] and its constructive variant [3]. In the case $G=\mathbb{R}$, the number $\mu(f)$ is also equal to $M(f)=\lim _{N \rightarrow \infty} \frac{1}{2 N} \int_{-N}^{N} f$ which is usually used in this theorem. See [6] (p.171) for a constructive proof of this fact. Classically, $M$ is an integral on the space of almost periodic functions. Constructively, the sum of two almost periodic functions need not be almost periodic, so $M$ can not be an integral on the set of all almost periodic functions. 


\section{Almost periodic functions on general topological groups}

In this section we extend the results from the previous section to arbitrary topological groups. Therefore, we let $G$ denote a topological group and $e$ denote its unit.

Definition 4. Let $f$ be a bounded continuous function on $G$. Define the operators $T_{s}:=$ $\lambda g \lambda x . g(s x)$ and $T^{s}:=\lambda g \lambda x . g(x s)$, for all $s \in G$. A function $f$ is left almost periodic if the set $S_{f}=\left\{T_{s} f: s \in G\right\}$ is totally bounded in $C_{b}(G)$, it is right almost periodic if the set $S^{f}=\left\{T^{s} f: s \in G\right\}$ is totally bounded in $C_{b}(G)$. Finally, $f$ is called almost periodic if it is both left and right almost periodic.

In the following it is often the case that the proof that $S^{f}$ is totally bounded is symmetric to the proof that $S_{f}$ is totally bounded. In such cases we will only prove the latter statement.

Lemma 1. Every almost periodic function $f$ is normable.

Proof. If $f$ is almost periodic, then so is $|f|$. Now, $f=\lambda s .\left(T_{s} f\right)(e)$ and hence $\|f\|=$ $\sup _{s \in G}|f(s)|=\sup _{g \in S_{|f|}} g(e)$ exists.

Every continuous function $f$ on a compact group $H$ is almost periodic. Indeed, $S_{f}$ is totally bounded, since it is the uniformly continuous image of the compact set $H$.

Let $f$ be almost periodic on $G$. Define the pseudo-metric

$$
d_{f}(a, b):=\sup _{g \in S_{f}}|g(a)-g(b)|
$$

on $G$. As before, the space $\left(G, d_{f}\right)$ is totally bounded, and its completion $G_{f}$ is a compact group. The function $f$ is continuous on $G_{f}$, and by the Peter-Weyl theorem $6 f=\sum_{\chi} f * \chi$ in $L_{2}\left(G_{f}\right)$; here the sum ranges over the character space $\Sigma$. For each character $\chi, T_{s}(f * \chi)=$ $\left(T_{s} f\right) * \chi$, so that $f * \chi$ is almost periodic. The function $f * \chi$ is even minimal almost invariant.

Definition 5. A function $f \in C(G)$ is called left almost invariant if the set $A$ of translations of $f, \operatorname{span}\left\{T_{s} f: s \in G\right\}$ is a finite-dimensional subspace of $C(G)$. It is called almost invariant if it is both left and right almost invariant.

It is called minimal almost invariant, if, moreover, every nonzero subspace of $A$ which is closed under the translations equals $A$.

To see that $f * \chi$ is almost invariant we recall from [3] that $\lambda f \cdot \chi * f$ is both a projection and a compact operator, so its range is finite dimensional. To see that it is minimal we need some preparations.

We consider $C\left(G_{f}\right)$ as a *algebra with the convolution operator * as multiplication and the map $\sim$ defined by $\tilde{f}:=\lambda x \cdot f\left(x^{-1}\right)$ as involution. Then $p$ is called a projection if $p=p * p=\tilde{p}$.

Lemma 2. 8] (p.216) A closed subspace in $L_{1}$ is a left(right) ideal in $L_{1}$ if and only if it is invariant under left(right) translation. The same holds for subspaces of $L_{2}$.

Lemma 3. Every nonzero closed ideal $I$ contains a nonzero central element.

Proof. Remark that $I^{*}:=\{\tilde{f}: f \in I\}$ is a right-ideal, so that $I^{*} I \subset I^{*} \cap I$. If $f \in I$ and $f \neq 0$, then $\tilde{f} * f \neq 0$, because $\tilde{f} * f(e)=\|f\|_{2}^{2} \neq 0$. Hence $I^{*} \cap I$ is a closed *subalgebra which contains a nonzero (self-adjoint) element $g$. Since $I$ is both a left and a right ideal it is closed under left and right translations, so the projection on the center $P_{Z} f:=\lambda x . \int f\left(z x z^{-1}\right) d z$ is also contained in this ideal. 
Since the only central elements in the ideal $I$ generated by a character $\chi$ are multiples of this character, we see that any nonzero closed subideal must actually be equal to $I$. It follows that $f * \chi$ is minimal almost invariant.

This proves the following theorem.

Theorem 9. Let $f$ be an almost periodic function on a topological group $G$. Then $f=$ $\sum f * \chi$ in $L_{2}\left(G_{f}\right)$ where the sum ranges over $\Sigma$. Moreover, each term $f * \chi$ is minimal almost invariant.

The following theorem is proved in a similar way as Theorem 8. It is a Bohr approximation theorem for general topological groups.

Theorem 10. Let $G$ be a topological group. Every almost periodic function on $G$ can be uniformly approximated by a linear combination of minimal almost invariant functions which is almost periodic.

\section{Conclusions}

We have given a constructive proof of the Bohr approximation theorem for general topological groups, thus simplifying and generalizing previous constructive approaches.

Finally, Loomis [6] proves that every left almost periodic function is also right almost periodic. His proof is non-constructive. To be precise consider his Lemma 41B. Let $n$ be the number of elements of the family $a_{i}$ and let $\underline{n}$ denote the finite set with $n$ elements. Then one needs to isolate all the functions $j: \underline{n} \rightarrow \underline{n}$ which correspond to a given $b \in G$. In this way we obtain a subset of a finite approximation to the space of translated functions. Classically, this approximation is totally bounded, but constructively one needs a finitely enumerable set. It is unclear to me whether this can be proved constructively.

Parts of this research can already be found in my $\mathrm{PhD}$-thesis [9]. I would like to thank Wim Veldman for his advice during this period. Finally, I would like to thank the referees for suggestions that helped to improve the presentation of the paper.

\section{REFERENCES}

[1] Errett Bishop and Douglas Bridges. Constructive analysis, volume 279 of Grundlehren der Mathematischen Wissenschaften. Springer-Verlag, 1985.

[2] J. Brom. The theory of almost periodic functions in constructive mathematics. Pacific Journal of Mathematics, 70:67-81, 1977.

[3] Thierry Coquand and Bas Spitters. A constructive proof of the Peter-Weyl theorem. Mathematical Logic Quarterly, 4:351-359, 2005.

[4] Fred Richman. Generalized real numbers in constructive mathematics. Indagationes Mathematicae, 9:595-606, 1998.

[5] C.G. Gibson. On the almost periodicity of trigonometric polynomials in constructive mathematics. Indagationes Mathematicae, 34:355-361, 1972.

[6] Lynn H. Loomis. An introduction to abstract harmonic analysis. University Series in Higher Mathematics. van Nostrand, New York, 1953.

[7] Margenstern, Maurice. On a variant of the constructive theory of almost periodic functions. Zeitschrift für Mathematische Logik und Grundlagen der Mathematik, 24:495-507, 1978.

[8] M.A. Naimark and A.I. Stern. Theory of group representations, volume 246 of Grundlehren der Mathematischen Wissenschaften. Springer-Verlag, 1982.

[9] Bas Spitters. Constructive and intuitionistic integration theory and functional analysis. PhD thesis, University of Nijmegen, 2002.

[10] S. Sternberg. Group theory and physics. Cambridge University Press, 1994.

[11] J. v. Neumann. Almost periodic functions in a group. I. Trans. Amer. Math. Soc., 36(3):445-492, 1934. 